$\underline{\underline{p}=\mathrm{m}}$

\title{
Pattern of occlusal contacts in intercuspal position of natural teeth
}

\author{
Tanzeela Imran $^{1 *}$, Naseer Ahmed ${ }^{2}$, Bushra Nazeer ${ }^{3}$ \\ ${ }^{1}$ Houseofficer B.D.S,Department Of Prosthodontics, Pakistan \\ ${ }^{2}$ Associate Professor B.D.S, FCPS Department Of Prosthodontics, Pakistan \\ ${ }^{3}$ Clinical Demonstrator,Department Of Prosthodontics, Pakistan \\ *Corresponding author E-mail:zeela_imran@yahoo.com
}

\begin{abstract}
The purpose of this study was to determine the patterns of occlusal contacts and location in intercuspal position of natural teeth. A cross-sectional study was conducted with a sample size of around seventy patients within a span of seven months, complete mouth impressions were made of each patient with hydrocolloid material and the impressions were poured in dental stone. The contact areas of the teeth in the maximum intercuspation position were determined by wax. Three distinct types of indentations were noted on the wax interocclusal record. Then similarly the centric occlusion contacts of the teeth on the cast were determined by interposing a new piece of wax, approximating the casts in the maximum intercuspation position by hand, and applying pressure. The oral and cast wax recordings were then compared. The occlusal relation for each individual tooth was then classified and recorded in a Performa.

Following wax interocclusal record of natural teeth in intercuspation an ideal occlusion was absent, a difference in morphology of anterior and posterior teeth was also evident that inevitably affects the nature of occlusal contacts.

The ideal occlusion was not found in any of the patient's investigation.
\end{abstract}

Keywords: intercuspation; interocclusal; ideal occlusion; natural teeth; occlusal contacts.

\section{Introduction}

Occlusion is defined as a manner in which the upper and lower teeth intercuspate between each other at all mandibular positions and movements. An ideal occlusion is a hypothetical or theoretical concept based on the anatomy of the teeth and is rarely found in nature. This concept is applied to a condition when the skeletal bases of maxilla and mandible are of the correct size relative to each other and the teeth should be in correct relationship in all three plane of space at rest. (Hassan \& Rahimah 2007, p.3-9) An ideal occlusion is achieved when the teeth are in maximum intercuspation position which refers to the occlusal relationship of the maxilla and the mandible in which the cusps of the teeth of both arches are fully interposed with each other. The teeth occlude in such a manner where there is maximum activity of the muscles and this is achieved by the shape and position of the teeth, periodontal fibres, muscle memory and occlusal contacts. (Kanno \&Abrão 2012, p.138-147)

The ideal patterns of tooth intercuspation have been described by Friel (Ash \& Ramjford 1982) for deciduous, mixed, and adult dentitions. He explained the location of the various contacts between the upper and lower teeth and divided the contacts into four categories: (1) cusp point contact with a fossa, a groove, or the occlusal embrasure between teeth, (2) ridge contact with an embrasure, (3) ridge contact with a groove and (4) surface contacts.

Several studies have been conducted describing the distribution of occlusal contacts at intercuspal positions in different populations, regarding the number, location and nature of contacts (Friel et al.1927; Stuart et al.1964; Beyron et al.1969; Ash \&Ramjford 1982) stating that the natural complete dentition is different from the ideal.
The rationale of our study was that the intercuspation of teeth plays an important role in restorative dentistry as the long term success of any restoration is dependent on the maintenance of occlusal harmony in order to fabricate a restoration that will not cause any occlusal discrepancy. Therefore our study will guide dental professionals in fabricating a restoration with the correct occlusal anatomy and in the alignment of teeth in dental procedures. Unsteadiness and alterations of occlusal contacts may lead to non-equilibrium situations, determining muscular stresses which lead to pathological occlusion.

\section{Materials and methods}

A sample size of seventy patients both males and females aged between 21 to 44 years were included in the study within a span of seven months at Altamash Institute of Dental Medicine Karachi, Pakistan. Patients included in the study had complete dentitions except a few in which third molars were absent. All patients had good oral hygiene, no periodontal disease, and fixed restorations. Complete mouth impressions were made of each patient with an irreversible hydrocolloid material and occlusal contact points at intercuspal position were recorded with a modeling wax interocclusal check record and stored in a moist ziplock bag.

Three different types of indentations were examined on the wax interocclusal record. The first type represented near contact, showed a depression with slight thinning of the wax. The second type represented contact, as it showed an indentation recognized by the creation of a translucent area by placing the wax bite in front of the light source. The third type represented supracontact formed by the perforation of the wafer. The latter two types were included in our study. 
The impressions taken were then poured with dental stone and centric occlusion contacts of the teeth on the casts were noted by applying pressure when placing a new wax sheet between the casts. The indentations on the wax bite obtained from the patient's mouth were visually compared with the wax record obtained through the approximation of casts. The two indentations coincided in location.

The comparison of the oral and cast wax recordings helped us to accurately record the locations of contact areas in both the maxillary and mandibular arches. The occlusal contact areas on the casts were located with the help of carbon paper. The contact areas recorded were evaluated and classified as the following types as stated by George E Meyers and Anderson in their study:

Type 1: No opposing tooth.

Type 2: No contact with an opposing tooth.

Type 3: Contact with a "flat plane" i.e. bottom of the fossa, marginal ridge, cusp tip.

Type 4: Contact on an inclined plane: i.e. cuspal incline or lingual or labial surface of an incisor.

Type 5: Contact with two or more converging inclined planes; e.g. planes on each side of a fossa.

Type 6: Combination of flat and inclined plane i.e. combination of 34 and 5 .

The data obtained were then entered in a Performa for future statistical analysis

\section{Results}

The 70 patients examined in this study had a total of 2106 teeth There were 1270 posterior teeth and 836 anterior teeth (Table II) Twenty nine patients had occlusions in which all the teeth made contact in centric occlusion.

The remaining 41 patients had from 1 to 08 teeth that did not contact in centric occlusion. The difference in morphology of the anterior and posterior occluding surfaces leads to a variation in the nature of occlusal contacts. Therefore it was feasible to evaluate and analyze the data separately of both teeth sets.

Out of 836 anterior teeth, 10 did not contact an opposing tooth in centric occlusion. A mandibular incisor in these subjects had no contact because the maxillary antagonist was missing (Type 1) Table III.

Out of the remaining 674 teeth, twelve anterior teeth had no contact in centric occlusion even though the opposing tooth was present (Type 2) Table V. There were six incisors and canines in which centric occlusion contacts were on flat surfaces (marginal ridges) (Type 3) Table VII. Two hundred and thirty four maxillary teeth made contact in centric occlusion with 231 mandibular teeth. In all instances, the labial margin of the incisal edge of the mandibular teeth contacted the lingual surface of the maxillary teeth All these contacts were, therefore, in the single incline plane (Type 4).

The data collected concerning the 1270 posterior teeth showed that 35 posterior teeth had no contact with an opposing tooth because the opposing tooth was missing (Type 1) Table IV. Majority of these 35 posterior teeth were third molars. Forty five posterior teeth had no contact with an opposing tooth even though the opposing tooth was present in the dental arch (Type 2) Table IV. Most of these teeth were third molars.

Three hundred and forty three posterior teeth had centric occlusion contacts on flat surfaces e.g fossa; marginal ridge and cusp tip majorly (Type 3) Table VIII. Nine teeth found in the posterior region of the mouth had contact in centric occlusion on one inclined plane only (Type 4) Table IX. Eight hundred forty two posterior teeth had centric occlusion contact areas on two cuspal inclines on each side of a fossa (Type 5) table X. Six hundred and ninety two posterior teeth combination contacts on cuspal inclines and flat surfaces, such as a fossa or marginal ridge, at the same time (Type 6) Table XI.
Table 1: Age Distribution N=70

\begin{tabular}{llllll}
\hline Variable & Range & Minimum & Maximum & Mean & Std. Deviation \\
\hline Age & 24 & 20 & 44 & 25 & 4.64 \\
\hline
\end{tabular}

Table 2: Descriptive Statistics Showing Total Number of Teeth N=70

\begin{tabular}{lccccc}
\hline & Minimum & Maximum & Sum & Mean & $\begin{array}{l}\text { Std. Devia- } \\
\text { tion }\end{array}$ \\
\hline $\begin{array}{l}\text { Anterior } \\
\text { teeth }\end{array}$ & 11.00 & 12.00 & 836 & 11.94 & 0.23 \\
$\begin{array}{l}\text { Posterior } \\
\text { teeth } \\
\text { Total }\end{array}$ & 12.00 & 20.00 & 1270 & 18.14 & 2.01 \\
\hline
\end{tabular}

Table 3: Representing Type 1 Anterior Teeth Occlusal Relation (No Opposing Teeth)

\begin{tabular}{lll}
\hline Type 1 & Frequency & Percent \\
\hline 0 & 60 & 85.7 \\
1 & 8 & 11.4 \\
2 & 2 & 2.9 \\
\hline
\end{tabular}

Table 4: Representing Type 1 Posterior Teeth Occlusal Relation (No Opposing Teeth)

\begin{tabular}{lll}
\hline Type 1 & Frequency & Percentage \\
\hline 0 & 38 & 54.3 \\
1 & 21 & 30.0 \\
2 & 10 & 14.3 \\
3 & 4 & 1.4 \\
\hline
\end{tabular}

Table 5: Representing Type 2 Anterior Teeth Occlusal Relation ( No Contact with an Opposing Tooth)

\begin{tabular}{lll}
\hline Type 2 & Frequency & Percentage \\
\hline 0 & 58 & 82.9 \\
1 & 7 & 10.0 \\
2 & 2 & 2.9 \\
3 & 1 & 1.4 \\
4 & 2 & 2.9 \\
\hline
\end{tabular}

Table 6: Representing Type 2 Posterior Teeth Occlusal Relation (No Contact with an Opposing Tooth)

\begin{tabular}{lll}
\hline Type 2 & Frequency & Percentage \\
\hline 0 & 31 & 52.0 \\
1 & 30 & 38.0 \\
2 & 8 & 8.0 \\
7 & 7 & 2.0 \\
\hline
\end{tabular}

Table 7: Representing Type 3 Anterior Teeth Occlusal Relation (Contact with a Flat Plane; Marginal Ridge)

\begin{tabular}{lll}
\hline Type 3 & Frequency & Percentage \\
\hline 0 & 64 & 91.4 \\
1 & 3 & 4.3 \\
2 & 3 & 4.3 \\
Total & 06 & \\
\hline
\end{tabular}

Table 8: Representing Type 3 Posterior Teeth Occlusal Relation (Contact with a Flat Plane; Marginal Ridge, Cusp Tip)

\begin{tabular}{lll}
\hline Type 3 & Frequency & Percentage \\
\hline 0 & 7 & 10.0 \\
2 & 10 & 7.1 \\
3 & 12 & 5.7 \\
4 & 24 & 11.4 \\
5 & 30 & 8.6 \\
6 & 138 & 32.9 \\
7 & 63 & 12.9 \\
8 & 56 & 10.0 \\
10 & 10 & 1.4 \\
Total & 343 & 100.0 \\
\hline
\end{tabular}

Table 9: Representing Type 4 Posterior Teeth Occlusal Relation (Contact on an Inclined Plane)

\begin{tabular}{lll}
\hline Type 4 & Frequency & Percent \\
\hline 0 & 67 & 95.7 \\
2 & 4 & 2.9 \\
5 & 5 & 1.4 \\
Total & 09 & \\
\hline
\end{tabular}


Table 10: Representing Type 5 Posterior Teeth Occlusal Relation (Contact with Two or More Converging Inclined Planes)

\begin{tabular}{lll}
\hline TYPE 5 & Frequency & Percentage \\
\hline 3 & 3 & 1.4 \\
7 & 21 & 4.3 \\
8 & 32 & 5.7 \\
9 & 18 & 2.9 \\
10 & 150 & 21.4 \\
11 & 11 & 1.4 \\
12 & 132 & 15.7 \\
13 & 169 & 18.6 \\
14 & 70 & 7.1 \\
15 & 105 & 10.0 \\
16 & 96 & 8.6 \\
17 & 17 & 1.4 \\
18 & 18 & 1.4 \\
Total & 842 & 100.0 \\
\hline
\end{tabular}

Table 11: Representing Type 6 Posterior Teeth Occlusal Relation (Combination of a Flat and Inclined Plane Contacts)

\begin{tabular}{lll}
\hline Type 6 & Frequency & Percent \\
\hline 5 & 15 & 4.3 \\
6 & 24 & 5.7 \\
7 & 49 & 10.0 \\
8 & 128 & 22.9 \\
9 & 27 & 4.3 \\
10 & 110 & 15.7 \\
12 & 180 & 21.4 \\
13 & 78 & 8.6 \\
14 & 14 & 1.4 \\
15 & 15 & 1.4 \\
16 & 16 & 1.4 \\
18 & 36 & 2.9 \\
Total & 692 & 100.0 \\
\hline
\end{tabular}

\section{Discussion}

The ideal occlusion in the current study was not seen as stated by our results which could be due to a number of factors such as attrition, periodontal disease, supernumerary teeth and disturbances in the eruption pattern caused by early loss of deciduous and permanent teeth within the subjects participated in the study.

There was an asymmetric distribution of occlusal contacts seen throughout the natural dentitions. The natural occlusal relations in cases where no opposing teeth i.e. Type 1 contact areas were mostly seen due to the absence of third molars, since in patients under 25 years of age the third molars were still undergoing active eruption.

There were dentitions in which the maxillary incisors had no contact with mandibular teeth i.e. Type 2, though opposing teeth were present. No contact areas were found due to orthodontic disturbances, as some maxillary or mandibular teeth were protruded or retruded leading to an insignificant number of occlusal contacts at intercuspal position this was in accordance to (Razdolsky \& Sadowsky 1989, p.181-182) they postulated that post orthodontic treatment a much higher number of contacts and near contacts are created in both anterior and posterior teeth at maximum intercuspation.

In our study the most striking finding was that the highest frequencies of occlusal contacts were found in premolars and molars in a combination of two or more converging cuspal inclined planes i.e. Type 5 contact areas. This suggests that mostly cusps are the dominant areas of occlusal contact as compared to fossas and marginal ridges these findings were similar to the study of (Jacob et al.1981 p.419-421)

Our study showed that on single inclined plane in posterior teeth contacts were most frequently made between maxillary first and second molars this was also stated in the study carried out by (Korioth et al.1990 p.206-210) while studying the number of occlusal contacts whereas in anterior single inclined planes were simply created with contact from the labial surface of the mandibular incisors to the lingual surfaces of maxillary incisor teeth.

The centric occlusion contacts on flat surfaces like on a marginal ridge and cusp tip i.e. Type 3 occlusal areas in premolars and mo- lars is also depended on much higher occlusal and oral forces to achieve maximum intercuspation of natural teeth as it is not achieved by the physiological forces of the lips, cheeks and tongue instead the functional movements of TMJ play a major role here, as the distribution of occlusal contacts vary in subjects with temporamandibular joint disorders. These results were further found in a survey confirming that a significant relationship exists between distribution of occlusal contacts and TMD by (R. Ciancaglini et al.2002 p.1082-1090)

The study also showed that premolars and molars have greater contact areas to bear occlusal load, thus have greater occlusal pressure than canines and incisors the same findings were also found by (Abe \& Nogami 2012 p.923-930)

\section{Conclusion}

Our study concluded that ideal occlusion was not present in any of the natural dentition. The findings stated that at maximum intercuspation point an asymmetric distribution of occlusal contact areas are created. These contact areas when studied concluded that it is the premolars and the molars that play the most significant part in achieving maximum intercuspation in natural dentition and the majority of these teeth occlude on a combination of flat and inclined planes.

The incisors mostly occlude in single inclined planes and very few teeth had cusp tip to cusp tip or marginal ridge occlusion. Thus the findings stated that occlusion is mostly maintained by the premolars and molars which are capable of achieving high occlusal stresses at maximum intercuspation. Therefore a dentist before planning to achieve a balanced dental occlusion should take into account the role of the premolars and molars.

\section{References}

[1] Ra Hassan and Rahimah AK b."Occlusion, malocclusion and method of measurements-an overview." Archives of Orofacial Sciences 2 (2007): 3-9.

[2] Watanabe-Kanno, Gustavo Adolfo, and Jorge Abrão. "Study of the number of occlusal contacts in maximum intercuspation before orthodontic treatment in subjects with Angle Class I and Class II Division 1 malocclusion." Dental Press Journal of Orthodontics $17.1 \quad$ (2012)138 147http://dx.doi.org/10.1590/S2176-94512012000100017.

[3] Anderson, J. Russell, and George E. Myers. "Nature of contacts in centric occlusion in 32 adults." Journal of dental research 50.1 (1971): 7-13. http://dx.doi.org/10.1177/00220345710500013201.

[4] Stuart, Charles E. "Good occlusion for natural teeth." The Journal of Prosthetic Dentistry 14.4 (1964): 716-724. http://dx.doi.org/10.1016/00223913(64)90207-0.

[5] Beyron, H. Optimal Occlusion. Dent. Clin. North Am. 1969; 13:537-554.

[6] Ash, Major M., and Sigurd Peder Ramfjord. An introduction to functional occlusion. WB Saunders Company, 1982.

[7] Ciancaglini, R., et al. "The distribution of occlusal contacts in the intercuspal position and temporomandibular disorder." Journal of oral rehabilitation $29.11 \quad$ (2002): $1082-1090 . \quad$ http://dx.doi.org/10.1046/j.13652842.2002.00941.

[8] Korioth, T. W. P. "Number and location of occlusal contacts in intercuspal position." The Journal of prosthetic dentistry 64.2 (1990): 206-210. http://dx.doi.org/10.1016/0022-3913(90)90180-K

[9] Razdolsky, Yen, Cyril Sadowsky, and Ellen A. BeGole. "Occlusal contacts following orthodontic treatment: a follow-up study." The Angle Orthodontist 59.3 (1989): 181-185.

[10] Ehrlich, Jacob, and Shlomo Taicher. "Intercuspal contacts of the natural dentition in centric occlusion." The Journal of prosthetic dentistry 45.4 (1981) 419-421. http://dx.doi.org/10.1016/0022-3913(81)90104-9.

[11] Korioth, T. W. P. "Number and location of occlusal contacts in intercuspal position." The Journal of prosthetic dentistry 64.2 (1990): 206-210. http://dx.doi.org/10.1016/0022-3913(90)90180-K.

[12] Ciancaglini, R., et al. "The distribution of occlusal contacts in the intercuspal position and temporomandibular disorder." Journal of oral rehabilitation $29.11 \quad$ (2002): 1082-1090. http://dx.doi.org/10.1046/i.13652842.2002.00941.X

[13] Abe, Y., et al. "Occlusal-supporting ability of individual maxillary and mandibular teeth." Journal of oral rehabilitation 39.12 (2012): 923-930. http://dx.doi.org/10.1111/joor.12008. 